\title{
Draft genome sequence of wild Prunus yedoensis reveals massive inter-specific hybridization between sympatric flowering cherries
}

Seunghoon Baek ${ }^{1+}$, Kyung Choi ${ }^{2 \dagger}$, Goon-Bo Kim ${ }^{1}$, Hee-Ju Yu ${ }^{3}$, Ara Cho ${ }^{1}$, Hoyeol Jang ${ }^{1}$, Changkyun Kim ${ }^{4}$, Hyuk-Jin Kim², Kae Sun Chang ${ }^{2}$, Joo-Hwan Kim ${ }^{4^{*}}$ and Jeong-Hwan Mun ${ }^{1 *}$

\begin{abstract}
Background: Hybridization is an important evolutionary process that results in increased plant diversity. Flowering Prunus includes popular cherry species that are appreciated worldwide for their flowers. The ornamental characteristics were acquired both naturally and through artificially hybridizing species with heterozygous genomes. Therefore, the genome of hybrid flowering Prunus presents important challenges both in plant genomics and evolutionary biology.

Results: We use long reads to sequence and analyze the highly heterozygous genome of wild Prunus yedoensis. The genome assembly covers $>93 \%$ of the gene space; annotation identified 41,294 protein-coding genes. Comparative analysis of the genome with 16 accessions of six related taxa shows that $41 \%$ of the genes were assigned into the maternal or paternal state. This indicates that wild $P$. yedoensis is an $\mathrm{F} 1$ hybrid originating from a cross between maternal P. pendula f. ascendens and paternal P. jamasakura, and it can be clearly distinguished from its confusing taxon, Yoshino cherry. A focused analysis of the S-locus haplotypes of closely related taxa distributed in a sympatric natural habitat suggests that reduced restriction of inter-specific hybridization due to strong gametophytic self-incompatibility is likely to promote complex hybridization of wild Prunus species and the development of a hybrid swarm.

Conclusions: We report the draft genome assembly of a natural hybrid Prunus species using long-read sequencing and sequence phasing. Based on a comprehensive comparative genome analysis with related taxa, it appears that cross-species hybridization in sympatric habitats is an ongoing process that facilitates the diversification of flowering Prunus.
\end{abstract}

Keywords: Flowering Prunus, Hybrid genome, Long-read sequencing, Sequence phase, S-locus haplotype

\section{Background}

Over the past several decades, genome analyses of diverse plant species have revealed that almost all plant genomes have experienced polyploidy events during their evolutionary history, suggesting that polyploidy has played an important role in plant diversification and

\footnotetext{
* Correspondence: kimjh2009@gachon.ac.kr; munjh@mju.ac.kr

†Seunghoon Baek and Kyung Choi contributed equally to this work.

${ }^{4}$ Department of Life Science, Gachon University, Seongnam 13120, Korea

'Department of Bioscience and Bioinformatics, Myongji University, Yongin 17058, Korea

Full list of author information is available at the end of the article
}

speciation (reviewed in [1]). Plant speciation that arises from polyploidy occurs via genome doubling within a species or through hybridization, either between closely related populations of the same species (autopolyploidy) or, more commonly, inter-specific or intergeneric hybridization (allopolyploidy). In both hybridization processes, generation of a heterozygous genome by hybridization can be a potential source of new species; the heterozygous filial generations may show different levels of heterosis or inbreeding depression due to variation between the homologous chromosomes. There are

(c) The Author(s). 2018 Open Access This article is distributed under the terms of the Creative Commons Attribution 4.0 International License (http://creativecommons.org/licenses/by/4.0/), which permits unrestricted use, distribution, and 
many examples of natural hybrid species reported from a wide range of monocots and dicots. In addition, inter-specific hybrid plants have been developed by breeding programs for agricultural or commercial purposes. Therefore, hybridization has been considered a creative force of evolution in plants [2]. Additionally, the sequencing and assembly of the genomes of hybrid plants presents an important challenge.

Plant genome sequencing has been facilitated by the introduction of next-generation sequencing (NGS) technologies that enable individual research groups to sequence and assemble the entire genome of interest. The sequencing and assembly of the genomes of plants, especially crop species, has typically been performed with homozygous or inbred lines using short-read sequencing; the resulting assemblies represent the homozygous haploid genome. In contrast, assemblies of heterozygous individuals, including natural tree species, require accurate handling of sequence reads to reconstruct the separate chromosome sets. For this reason, heterozygous plant genomes still pose considerable challenges in genome sequencing and assembly. To overcome the weaknesses of short-read assemblies, which cannot resolve a mixture of highly similar sequences such as duplicated genes or repetitive transposons [3], long-read sequencing technology, such as PacBio sequencing, is a good choice because long-read data can be phased into individual chromosomes during assembly of the reads [4]. For example, an Arabidopsis F1 hybrid genome and a heterozygous grapevine accession genome were successfully de novo assembled into haplotigs using a FLACON assembler and PacBio reads [5]. Therefore, long-read sequencing technology combined with relevant assembly algorithms could help the assembly of the heterozygous genomes derived from hybridization.

Rosaceae is a flowering plant family consisting of approximately 3000 species in 90 genera and large number of inter-specific and intergeneric hybrids [6]. The rose family includes diverse plant species such as herbs, shrubs, and trees that are widespread in northern temperate regions. A number of species are economically important as food crops that produce fruits and nuts. Due to their economic and agronomic importance, the genomes of several Rosaceae fruit crop species have been sequenced, including the domesticated apple [7], sweet cherry [8], Chinese plum [9], peach [10], pear [11, $12]$, and strawberry [13, 14]. Ornamental species (rose, flowering cherry, hawthorn, etc.) are also grown for gardening and residential landscape purposes. In particular, roses in genus Rosa and cherry blossom trees in genus Prunus are popular plants worldwide for their beautiful blossoms and superior ornamental characteristics. Genus Prunus is a member of tribe Amygdaleae that develops a drupe. Despite the large number of Prunus species (approximately 250), the majority of flowering cherry species are originally native to eastern Asia, including Korea, Japan, and China, where various natural and artificial hybrids have been developed and selected $[15,16]$. Due to a long history of cultivation along with naturalization of wild flowering cherry species and inter-specific hybridization in eastern Asia, there has been confusion over name, origin, and delimitation between taxa.

One of the controversial issues raised in flowering Prunus species is the relationship between cultivated and wild taxa of $P$. yedoensis and their relatives. Among the diverse flowering Prunus species, the "Yoshino cherry" tree $(P . \times$ yedoensis, Pxy) from Japan is one of the most popular hybrid species that has been extensively planted not only in Japan, but also in many other locations, for example, the Tidal Basin in Washington, DC, USA. The "Yoshino cherry" was derived from a cross between paternal $P$. speciosa and maternal $P$. pendula f. ascendens (Ppa) [17]. Meanwhile, wild P. yedoensis (P. yedoensis var. nudiflora Koehne [Pyn]), which is referred to as the wild "King cherry" and has superior flower, cherry, and shape ornamental characteristics, is endemic to Jeju Island, Korea. It was first discovered in 1908 in the natural habitat of Mt. Halla on Jeju Island [18]; several natural populations have been conserved as a National Monument of Korea. Due to its scientific name, there has been confusion about whether the wild "King cherry" and the "Yoshino cherry" are the same taxa or if the "Yoshino cherry" originated from wild P. yedoensis. Phylogenetic studies using a limited number of DNA markers suggested that Pyn is closely related to the "Yoshino cherry," but that these two taxa are distinguishable [19, 20]. However, a genome-level comparison between the two taxa has not been reported so far.

Prunus species have a gametophytic self-incompatibility (GSI) system to avoid self-fertilization. The interaction between pollen and the style or ovule of Prunus species is determined by a specific pair of S-locus genes, a male determinant $S$ haplotype-specific F-box protein (SFB) and a female determinant S-locus ribonuclease (S-RNase). The two loci are completely linked to maintain the co-evolved allele specificities for the compatibility of male and female gametes; therefore, the S-RNase and SFB gene pair is referred to as the S haplotype in Prunus [21, 22]. Similar to the case of Pxy, Pyn is also likely to be a natural hybrid derived from maternal Ppa and an unknown paternal Prunus species [23], suggesting compatibility of the $\mathrm{S}$ haplotype between distinct Prunus species. Recently, we investigated the genetic structure of the Pyn population along with candidate parental Prunus species on Jeju Island using 20 nuclear gene-based DNA markers and found that Pyn may originate from a cross between maternal Ppa and paternal $P$. jamasakura (Pj). In addition, approximately $81 \%$ 
of the wild Pyn accessions examined were likely F1 hybrids, whereas the remaining 19\% were backcross hybrids resulting from additional asymmetric introgression of parental genotypes, suggesting that Pyn on Jeju Island is a homoploid inter-specific hybrid [24]. However, there are still additional questions that need to be answered, including the organization of the Pyn genome as a homoploid hybrid and how the putative parental genomes hybridized into the Pyn genome. These concerns can be addressed by a comprehensive and in-depth analysis of the Pyn genome.

With the aim of describing the hybrid genome, which is fundamental for understanding the structure and organization of the hybrid flowering Prunus genome, in this study, we report the draft genome assembly, annotation, and analysis of the heterozygous wild Pyn genome based on PacBio RSII long-read sequencing refined with Illumina short-read sequences. We assembled the Pyn genome into "haplotype-fused" long contigs then inferred haplotypes of genic regions by mapping to the short-read sequences of putative parental species. We also conducted whole-genome resequencing analysis of five Pyn accessions and 11 accessions of related Prunus species to verify the parental origin and genomic delimitation of hybrid taxa. Comprehensive analysis of heterozygous genome assembly and variation data between genotypes collectively provides novel insights into the organization and hybridization of the wild flowering Prunus genomes.

\section{Results}

Highly heterozygous genomic nature of wild $P$. yedoensis Pyn-Jeju2 is an endemic wild flowering cherry tree preserved in a natural habitat on Jeju Island, Korea with a beautiful shape, pink flowers, and black berries (Fig. 1a). The nuclear genome of Pyn is organized into eight chromosomes $(2 n=2 x=16)$; the size and structure of Pyn chromosomes are highly similar to those of Ppa and $P$. jamasakura var. jamasakura (Pji) [25]. The genomic characteristics of Pyn-Jeju2 were viewed through substring of length $\mathrm{K}$ (K-mer) analysis using Illumina short reads at a K-mer size of 17 . As shown in Fig. 1b, the frequency distribution of K-mer showed two clear peaks located at coverage $48 \times$ and coverage $98 \times$, corresponding to the heterozygous and homozygous reads, respectively. The heterozygous read peak showed a frequency approximately twofold that of the homozygous read peak, demonstrating that Pyn-Jeju2 has a hybrid genome. The maximum haploid genome size of Pyn was estimated to be 257 megabases $(\mathrm{Mb})$ based on homozygous reads. Moreover, the heterozygous fraction of the Pyn genome predicted that the maximum diploid genome size of Pyn is $525 \mathrm{Mb}$. A flow cytometry assay also estimated a similar haploid genome size of $284 \mathrm{Mb}(1 \mathrm{C}=0.29)$, which was in accordance with the range of diploid Prunus genomes (mean $1 \mathrm{C}=0.28$ ) reported in the Plant DNA $\mathrm{C}$-value database [26]. Additional K-mer analysis of four Pyn accessions and four "Yoshino cherry" accessions showed fundamentally similar patterns of K-mer frequency distribution (Additional file 1: Figure S1). These findings collectively suggest that Pyn is a natural homoploid hybrid.

\section{De novo genome assembly of wild $P$. yedoensis}

The main challenge of the Pyn genome for de novo assembly is its high heterozygosity. Considering the hybrid genome structure of Pyn, we applied PacBio RSII
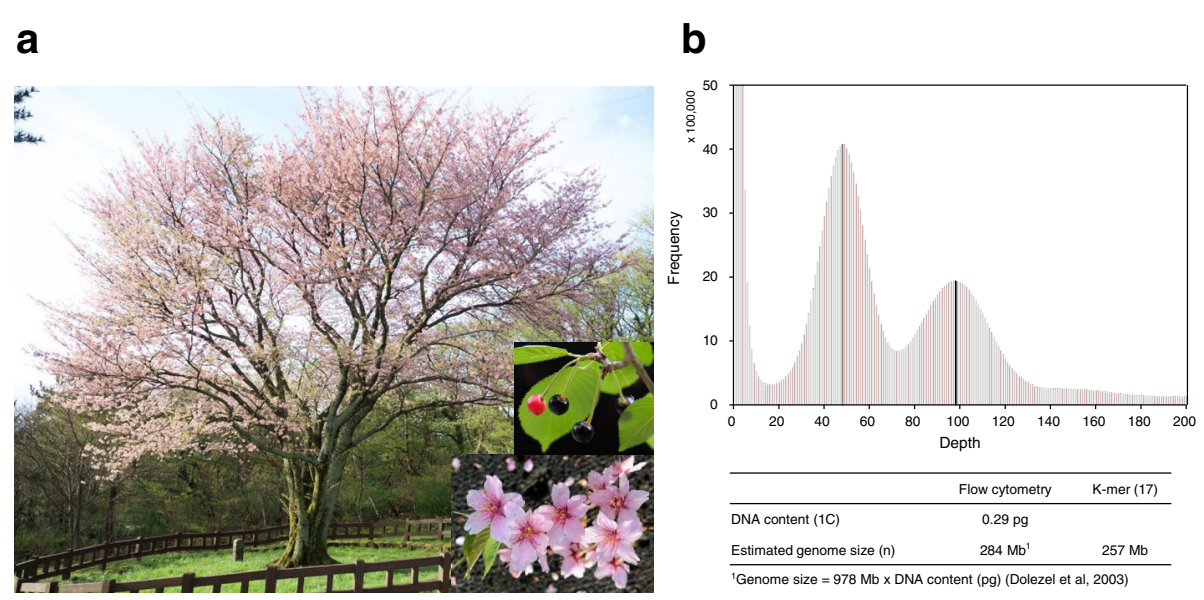

Fig. 1 The reference accession of wild Prunus yedoensis used in this study. a Photographs of a Pyn-Jeju2 tree and its flowers and berries taken from March to April 2017. b Estimation of the genome size of Pyn-Jeju2 based on K-mer analysis. The top panel represents the volume of K-17mer (Y-axis) plotted against the frequency at which it occurs (X-axis). The gray and black peaks correspond to heterozygous and homozygous reads, respectively. The bottom panel shows the estimated haploid genome size based on the homozygous K-mer peak as well as flow cytometry analysis 
sequencing and the FALCON assembler as a long-read sequencing and overlap-layout-consensus assembly strategy (Additional file 1: Figure S2). We generated approximately 18.8 gigabases (Gb) of PacBio RSII reads affording 73-fold coverage of the haploid genome. In addition, 306.6 Gb of Illumina short-read sequences were also generated for correction of the PacBio reads, additional assembly, and scaffolding (Additional file 2: Table S1). After read correction and quality trim, filtered sequences $(85.0 \mathrm{~Gb})$ were de novo assembled into 4292 contigs (N50 of 132.6 kilobases [kb]), as well as a complete chloroplast genome that was identical to the previously reported sequence (GenBank accession NC_026980). The initial assembly included both "haplotype-fused" contigs and associate contigs representing highly divergent regions between the homologous sequences. Additional assembly with Illumina mate-paired (MP) reads and Fosmid-end sequences allowed for the generation of the Pyn draft genome assembly, which consisted of 3185 scaffolds (Table 1). The Pyn draft genome assembly was $323.8 \mathrm{Mb}$ (scaffold N50 of $199.0 \mathrm{~kb}$ ), which was $126.0 \%$ of the estimated haploid genome size. Comparing this assembly to transcriptome data revealed that the draft assembly can recover $>93 \%$ of the gene space (Additional file 3: Table S2; Additional file 4: Table S3). Analysis of variation based on single nucleotide polymorphisms (SNPs) identified by mapping of Illumina short reads on to the assembly showed that $2.5 \mathrm{Mb}(1.1 \%)$ of the total contig length was classified as heterozygous.

\section{General features of the wild $P$. yedoensis genome}

The statistics of repetitive sequences and protein-coding genes in the Pyn genome are depicted in Table 2, Additional file 5: Table S4, Additional file 6: Table S5, and Additional file 7: Table S6. Sequence analysis of the draft genome assembly showed that the overall proportion of repetitive sequences as well as the number of protein-coding genes were higher in Pyn and sweet cherry ( $P$. avium, Pa) than in Chinese plum ( $P$. mume,

Table 1 Summary statistics of the draft genome assembly of wild $P$. yedoensis

\begin{tabular}{llllll}
\hline & Contig & & & \multicolumn{2}{l}{ Scaffold } \\
& Length $(\mathrm{bp})$ & Number & & Length (bp) & Number \\
\hline N90 & 38,284 & 2435 & & 54,586 & 1700 \\
N80 & 59,290 & 1770 & & 88,524 & 1239 \\
N70 & 81,939 & 1312 & & 124,582 & 934 \\
N60 & 106,886 & 973 & & 158,837 & 702 \\
N50 & 132,585 & 706 & & 198,954 & 519 \\
Longest & 773,088 & & & 960,226 & \\
Overall $(>1 \mathrm{~kb})$ & $318,739,121$ & 4292 & & $323,781,369$ & 3185 \\
\hline
\end{tabular}

Pm) and peach ( $P$. persica, $\mathrm{Pp})$ primarily due to an excessive amount of assembled sequence. Approximately $47.2 \%$ of the assembled Pyn genome consisted of repetitive sequences, with $24.9 \%$ retrotransposons and $14.9 \%$ DNA transposons. Similar to the cases of $\mathrm{Pa}, \mathrm{Pm}$, and $\mathrm{Pp}$, the most dominant retrotransposon in the Pyn genome was Ty3/Gypsy followed by Ty1/Copia, whereas CMC-EnSpm was the most abundant DNA transposon (Additional file 5: Table S4). In total, 41,294 protein-coding genes and 2187 RNA genes were predicted from the draft genome of Pyn (Table 2), which is 1.3 - to 1.5-fold more genes than the $\mathrm{Pm}$ and $\mathrm{Pp}$ genomes but slightly fewer genes than the Pa genome. However, the average length of protein-coding genes was shorter than those of the sequenced Prunus genomes due to a reduction of exon length. In contrast, the average gene density was $7.7 \mathrm{~kb}$ per gene, which is similar to Pm (7.6 kb per gene) but higher than $\mathrm{Pp}(8.2 \mathrm{~kb}$ per gene) and lower than $\mathrm{Pa}(6.2 \mathrm{~kb}$ per gene). Therefore, it is likely that overall genomic organization characteristics of Pyn are similar to those of fruit crop Prunus species. Of the predicted genes, 33,802 (81.9\%) had at least one match to messenger RNA sequencing (mRNA-seq) reads from the present study. Functional annotation identified 37,444 genes as "known" based on expression, database matches, or any detectable domain signatures, whereas the remaining 3850 (9.3\%) were assigned as "unknown" or "hypothetical" (Additional file 7: Table S6). Expression analysis of protein-coding genes revealed that a total of 4287 genes were alternatively spliced in different tissues and that 230 isoforms were tissue-specific alternative splicing variants. The most abundant types of alternative splicing events were alternative transcription start or termination sites (Additional file 8: Table S7).

Since the draft Pyn genome was assembled into "haplotype-fused" scaffolds as well as variant sequences that exceed the estimated haploid genome size, we could phase the gene models by mapping the short-read sequences of candidate parental species, Ppa (Ppa-1) and Pjj (Pji-1), even though the FALCON Unzip algorithm was unavailable when the draft genome was assembled (Fig. 2a). Phasing of genes based on SNP analysis with parental sequences showed that approximately $59.2 \%$ of genes were encoded in the homozygous sequence fraction and the remaining $40.8 \%$ of total genes were phased into either maternal $(19.4 \%)$ or paternal $(21.4 \%)$ origin (Table 3). Although Pyn-Jeju2 is a wild accession and none of its genetic resources, including a genetic map, were available, we tentatively assigned and ordered the scaffolds to the chromosome sequences of $\mathrm{Pp}$ as reference using BLAST and MCScanX [27] comparisons. The chromosome-assigned Pyn sequences covered 281.6 Mb, which included $87.0 \%$ of the draft assembly, and showed one-to-one chromosome level syntenic matches with $\mathrm{Pp}$ 
Table 2 Comparison of repetitive sequences and annotated protein-coding genes in the draft assemblies of four Prunus genomes

\begin{tabular}{|c|c|c|c|c|c|}
\hline Genome & Characteristics & P. yedoensis var. nudiflora & P. avium & P. mume & P. persica \\
\hline Draft sequences & Size (Mb) & 318.7 & 272.4 & 237.2 & 227.2 \\
\hline \multirow[t]{6}{*}{ Repetitive sequences } & No. RNA genes ${ }^{a}$ & 2187 & 729 & 1541 & 1243 \\
\hline & DNA TE (Mb) & 47.6 & 26.5 & 25.5 & 38.7 \\
\hline & RNA TE (Mb) & 79.5 & 56.4 & 49.9 & 62.6 \\
\hline & Simple repeats $(\mathrm{Mb})$ & 5.9 & 5.3 & 4.2 & 4.1 \\
\hline & Other repeats (Mb) & 5.6 & 3.9 & 3.3 & 2.7 \\
\hline & Total non-redundant bases (Mb) & 150.8 & 103.9 & 71.1 & 88.0 \\
\hline \multirow[t]{6}{*}{ Protein-coding genes } & Total number & 41,294 & 43,673 & 31,390 & 27,864 \\
\hline & Avr. gene size (bp) & 2154 & 2294 & 2514 & 2607 \\
\hline & No. exons per gene & 4.3 & 3.6 & 4.6 & 5.1 \\
\hline & Avr. exon size (bp) & 220 & 248 & 249 & 243 \\
\hline & Avr. intron size (bp) & 362 & 417 & 380 & 317 \\
\hline & Avr. gene density (kb/gene) & 7.7 & 6.2 & 7.6 & 8.2 \\
\hline
\end{tabular}

Statistics for P. avium, P. mume, and P. persica are based on Shirasawa et al. [8], Zhang et al. [9], and The International Peach Genome Initiative [10], respectively, and repetitive sequences were recalculated using the same criterion used to annotate the $P$. yedoensis var. nudiflora genome

${ }^{\text {a }}$ Sequences encoding ribosomal RNA, transfer RNA, and microRNA were considered
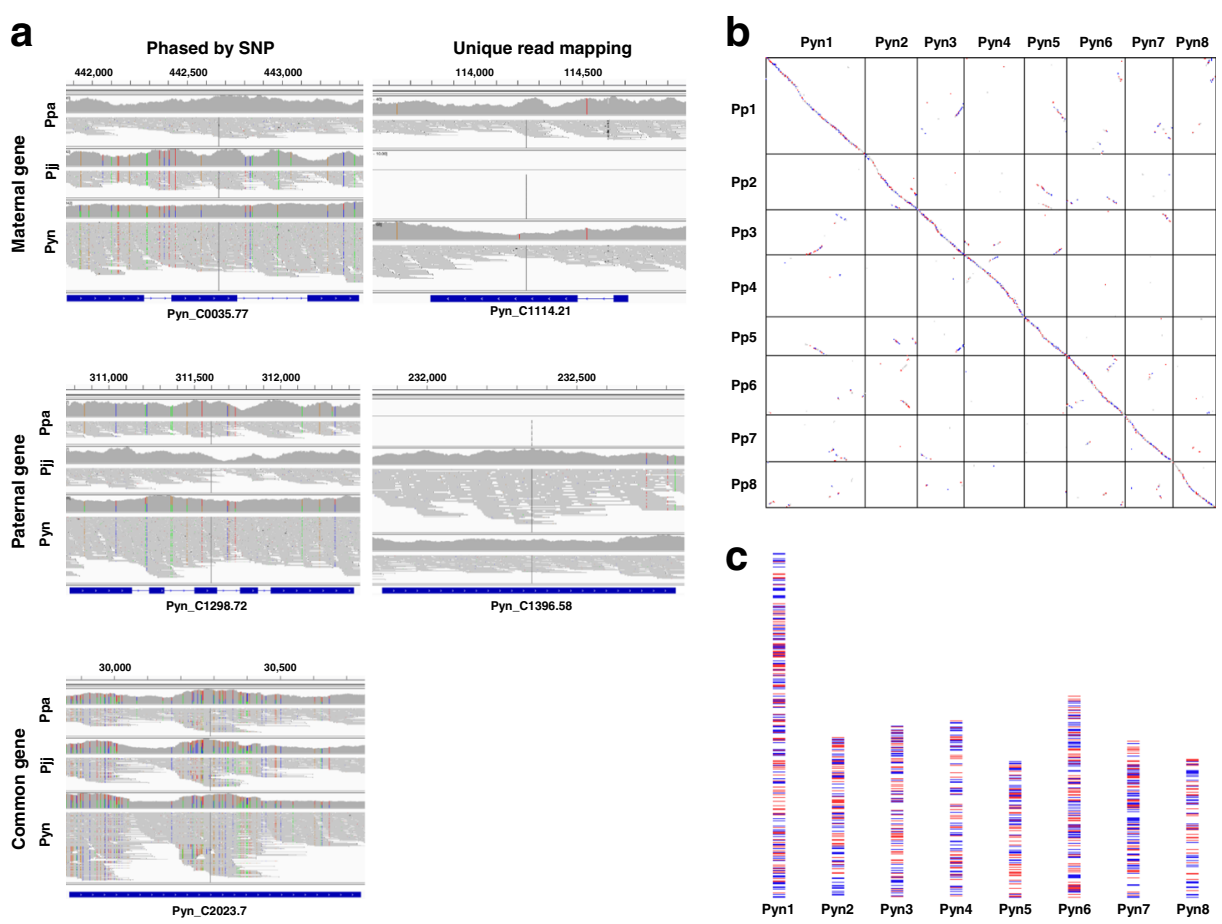

Fig. 2 Phasing and arrangement of the heterozygotic genome assembly. a Examples of haplotype-phased gene models. Gene models predicted from the initial "haplotype-fused" assembly are phased according to read mapping and SNP analysis using the Illumina short-read sequences of putative parental species. Genes were phased into one parental haplotype if a gene was aligned only by reads from one parental species (unique mapping) or had at least twofold as many supports for SNPs by reads of one parental species (phased by SNP). Genes with similar supports of read mapping for both parental species are defined as common type. Colored dots denote SNPs identified in the aligned reads. $\mathbf{b}$ Chromosomal arrangement of the gene-phased genome assembly of wild $P$. yedoensis (Pyn) onto the P. persica (Pp) genome. c Distribution of haplotypephased genes in the tentative chromosomes of wild P. yedoensis. Colored dots or lines represent maternal-phased genes (red), paternal-phased genes (blue), or common genes (gray) 
Table 3 Classification of wild $P$. yedoensis genes based on sequence phasing of the draft assembly by mapping of Illumina short-read sequences from putative parental species, maternal $P$. pendula f. ascendens, and paternal $P$. jamasakura

\begin{tabular}{|c|c|c|c|c|c|}
\hline \multirow[t]{2}{*}{ Type } & \multicolumn{2}{|c|}{ Maternal gene } & \multicolumn{2}{|c|}{ Paternal gene } & \multirow{2}{*}{$\begin{array}{l}\text { Common } \\
\text { gene }\end{array}$} \\
\hline & Unique & Phased & Unique & Phased & \\
\hline Number & 548 & 7482 & 1353 & 7456 & 24,455 \\
\hline Ratio (\%) & 1.3 & 18.1 & 3.3 & 18.1 & 59.2 \\
\hline
\end{tabular}

(Fig. 2b), Pa, and Pm genomes (Additional file 1: Figure S3). The chromosome-assigned sequences of Pyn covered $54.5 \%-66.4 \%$ of the sequenced Prunus genomes (Additional file 9: Table S8). The distribution of phased genes along the tentative Pyn chromosomes demonstrated a complicated mosaic pattern (Fig. 2c). These findings indicate that Pyn has a hybrid genome derived from $\mathrm{Ppa}$ and $\mathrm{Pjj}$ where parental sequences are organized into a hybrid genome with a random arrangement.

\section{Comparative genome analysis of wild $P$. yedoensis}

Comparative analysis of the Pyn genome revealed unique characteristics of the flowering cherry genome. A six-way comparison of Pyn, Pa, Pm, Pp, strawberry (Fragaria vesca, Fv), and apple (Malus $\times$ domestica, Mxd) genes using OrthoMCL analysis yielded 16,777 Pyn gene families (30,478 genes), of which 9221 (55.0\%) were shared by all six species. However, the analysis revealed 678 gene families (4.0\%) consisting of 1723 genes that were unique to Pyn (Fig. 3a). Gene annotation revealed 273 gene families (650 genes) as "known" with the remaining 405 gene families (1073 genes) assigned as "unknown" or "hypothetical." Comparison of gene families with the fruit crop Prunus genomes identified specific over-represented or under-represented $(p<0.001)$ gene families in the Pyn genome. For example, the P-loop-containing nucleoside triphosphate hydrolase superfamily genes and the $\mathrm{C} 3 \mathrm{HC}$ zinc finger-like genes were enriched, whereas the glycosyl hydrolase family and the zinc ion binding protein genes were under-represented in the Pyn genome (Additional file 10: Table S9). Of particular interest, NAC transcription factors (TF), auxin response factors, SNF2/Brahma-type chromatin remodeling protein, and plant neutral invertase genes were almost twofold more abundant in the Pyn genome compared to other fruit crop Prunus species. In addition, FLOWERING WAGENINGEN and Early Flowering 6 TFs that regulate flowering time were also abundant in the Pyn genome.

Divergence of the Pyn genome from the tribe Potentilleae (Fv), Maleae (Mxd), and fruit crop Prunus in subgenus Amygdalus (Pp), Prunus (Pm), and Cerasus (Pa) genomes was deduced based on the synonymous substitution rate (Ks) of homologous genes and Bayesian evolutionary analysis. As shown in Fig. 3b, splitting of the Pyn genome from its close relatives was conducted by comparing orthologous genes. Pyn shared a single peak with $\mathrm{Fv}$ and Mxd at Ks modes of 0.48 and 0.35,
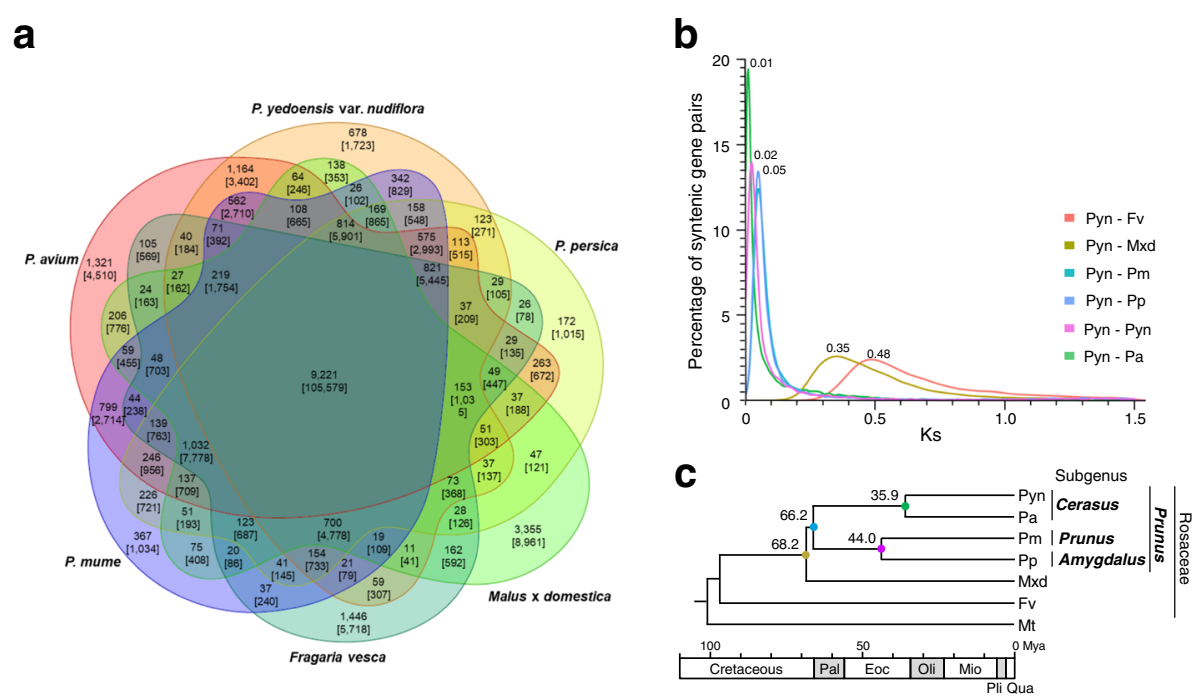

Fig. 3 Comparative analysis of the wild P. yedoensis var. nudiflora genome. a Venn diagram showing the unique and shared gene families between six sequenced genomes of the Rosaceae family. The number of gene families and genes (in bracket) for each group are shown. b Distribution of Ks values obtained from comparisons of orthologous gene sets between six genomes of the Rosaceae family and paralogous gene sets in wild $P$. yedoensis. c Genome evolution of Prunus species. Estimation of dates for speciation events are given in millions of years based on Bayesian evolutionary analysis of 276 conserved single copy genes. Pyn $P$. yedoensis var. nudiflora, Fv Fragaria vesca, Mt Medicago truncatula, Mxd Malus $\times$ domestica; Pa P. avium, Pm P. mume, Pp P. persica. Pal Paleocene, Eoc Eocene, Oli Oligocene, Mio Miocene, Pli Pliocene, Qua Quaternary 
respectively, indicating successive splitting of the Prunus lineage from Potentilleae and Maleae presumably during the Cretaceous and Paleocene periods around 88 to 61 million years ago (Mya) [28]. The Ks distribution between Prunus genomes showed a very recent diversification of Prunus species. The peaks at a Ks mode of 0.05 for orthologs between Pyn-Pm and Pyn-Pp genomes were essentially identical. Moreover, paralogs of the Pyn genome and orthologs between Pyn-Pa showed a single peak at Ks modes of 0.02 and 0.01 , respectively, demonstrating successive splitting of the Pyn genome from subgenera Amygdalus (Pp), Prunus (Pm), and Cerasus (Pa). Bayesian evolutionary analysis of 276 single copy orthologous genes conserved in six Rosaceae species and one outgroup Fabaceae species (Medicago truncatula, Mt) identified 376,758 aligned positions with 127,606 (33.7\%) variable sites, 29,134 (7.7\%) of which were parsimoniously informative. Figure $3 \mathrm{c}$ shows a chronogram estimating divergence time. Molecular dating analysis performed with BEAST estimated the age of genus Prunus at approximately 66.2 Mya (95\% higher posterior densities [HPD] of 64.2-67.4 Mya). Similarly, the age estimate for the split of subgenera Prunus and Amygdalus was around 44.0 Mya (95\% HPD of 42.2-45.6 Mya) and the divergence time between Pyn and Pa was estimated to be around 35.9 Mya (95\% HPD of 34.4-37.3 Mya). These results were consistent with a previous report on the rapid diversification of Prunus lineage of eastern Asian origin, presumably 35 Mya [28].

\section{Expression of lineage-specific genes in the inter-specific hybrid genome}

In hybrid organisms, genes are inherited from the two parents; differences in gene expression or modification may result in hybrid vigor or weakness. To determine the expression characteristics of the genes originating from a single parental lineage, we performed mRNA-seq analysis and expression profiling in five tissues, namely, leaf, petal, pistil, stamen, and berry (Additional file 3: Table S2). Overall, genes inherited from only one parental lineage (86.5\%) were more abundantly expressed than those from both parental lineages (78.6\%). Moreover, approximately $7.0 \%$ (562) of maternal genes and 6.5\% (576) of paternal genes were differentially expressed in various tissues (Fig. 4a). Most differentially expressed genes (DEGs), except for two maternal genes and two paternal genes, were expressed in more than two tissues, demonstrating less tissue specificity. Clustering analysis and a
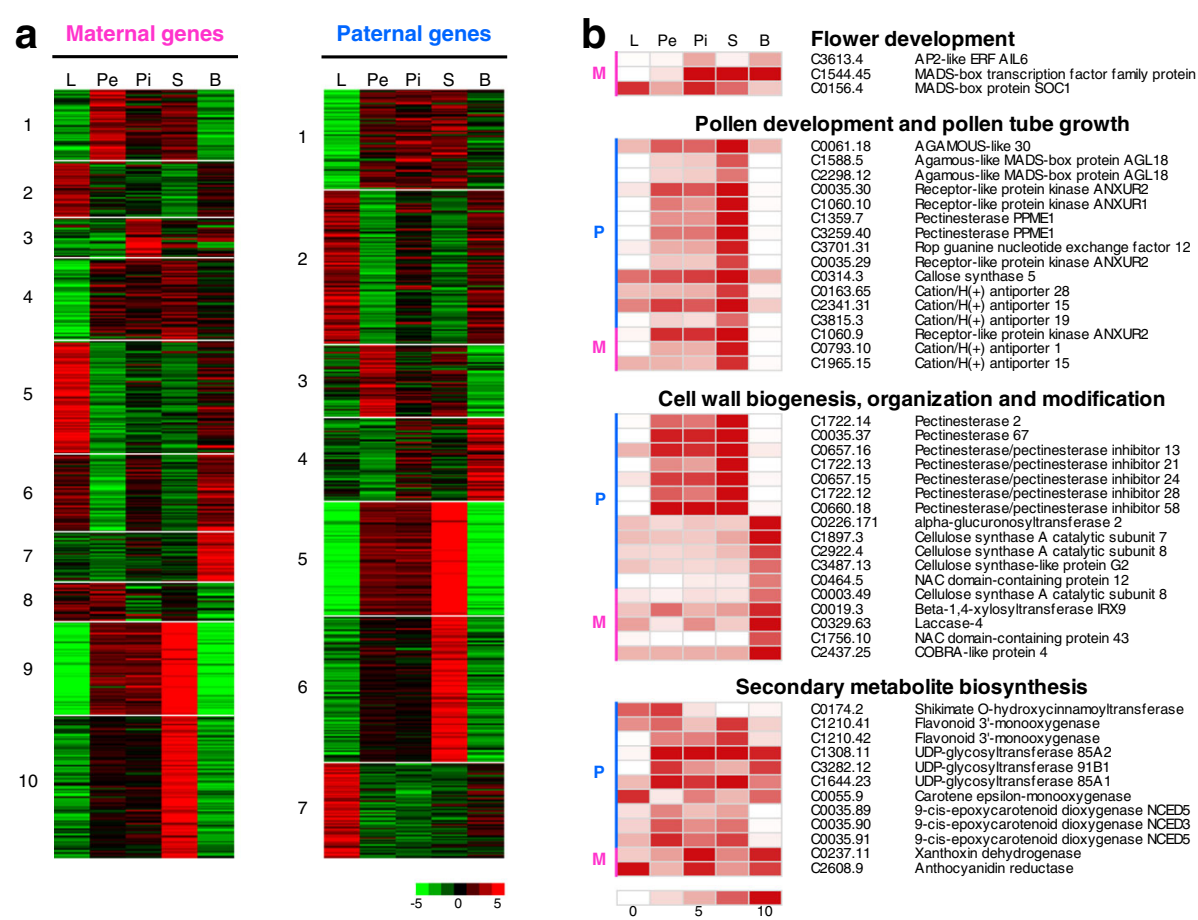

Fig. 4 Differential expression of haplotype-phased genes in various tissues of wild $P$. yedoensis. a Heat maps representing the expression of 562 maternal- and 576 paternal-phased genes, which are identified as differentially expressed genes in the mRNA-seq analysis, in different tissues. The normalized count values of a given gene from three independent biological replicates across all samples were used as a normalization factor. The vertical axes organize genes according to co-expression. The horizontal axes represent five tissues: leaf (L), petal (Pe), pistil (Pi), stamen (S), and berry (B). b Heat maps showing the differential expression of a selected category of genes related to development and secondary metabolite biosynthesis. The average normalized count values represent the relative expression across tissues. M maternal-phased genes, P paternal-phased genes 
functional enrichment study of DEGs for each parental lineage also showed that several gene groups that originated from only one parental lineage were outnumbered and expressed differentially in specific tissues (Fig. 4b). For example, three flower development-related TF (AP2-ERF and MADS-box TFs) genes that originated from the maternal lineage were differentially expressed in vegetative and reproductive tissues; however, no DEGs from the paternal lineage were identified. Meanwhile the paternal lineage had 2.5- to 5-fold more DEGs for groups of genes related to pollen or pollen tubes, the cell wall, and secondary metabolite biosynthesis than those from the maternal lineage. Regardless of the parental origin, these genes were more abundantly expressed in the three floral and berry tissues than in the leaf tissue.

\section{Resequencing analysis of wild $P$. yedoensis and its related species}

To study the genomic relationship between Pyn and its closely related species, we resequenced five Pyn accessions, four "Yoshino cherry" accessions (Pxy), and seven accessions of candidate parental species, including Ppa, $\mathrm{Pj}$ (Pjj and P. jamasakura var. quelpaertensis [Pjq]), and $P$. sargentii (Psa), and compared this resequencing data with the reference assembly of Pyn-Jeju2 (Table 4; Additional file 11: Table S10). The paired-end (PE) reads of Pyn-Jeju2 itself covered 99\% of its reference assembly. In addition, at least $91 \%$ of PE reads from other examined Prunus species were successfully mapped onto the reference genome of Pyn, covering $82-96 \%$ of the genome. This excludes Ppa-2 and Pjj-2, for which the genomes were sequenced at low coverage $(7.5 \times)$. Read mapping rate and reference genome coverage were the highest for Pyn genotypes, followed by Pxy and parental taxa.

In total, 76,427,804 SNPs and insertions or deletions (InDels) were identified by multi-sample variome analysis of all 16 resequencing samples (Table 4). Since all the accessions were wild accessions with heterozygous genomes (Additional file 1: Figure S1), the heterozygous SNP rate was approximately $52 \%$ of the total variome. Pxy accessions showed the most diverse genotypes with 6.3 million SNPs/InDels on average. In contrast, parental accessions had less variation (3.3 million variations for maternal and 4.2 million variations for paternal taxa on average) than Pyn accessions (4.9 million variations on average). There was no significant difference in variome size between the paternal taxa, Pjj, Pjq, and Psa. The transition/transversion $(\mathrm{Ti} / \mathrm{Tv}$ ) ratio in Prunus species was $1.52-1.60$. Interestingly, the Pyn-Jeju5 accession had 6.1 million variations, which was a similar level to Pxy accessions. Multidimensional scaling (MDS) and a maximum likelihood (ML) tree based on variome data indicated that the Pyn accessions were distinctly separated from the Pxy accessions, except for Pyn-Jeju5 (Fig. 5).
The Pyn-Jeju 5 accession was grouped together with the Pxy accessions on both the MDS plot and the ML tree, showing that this accession is more closely related to Pxy. Of particular interest, the Pyn accessions were located in the middle of maternal Ppa accessions and paternal $\mathrm{Pj}$ and Psa accessions by both dimensions of MDS, demonstrating that the Pyn accessions have intermediate genomic characteristics of the two parental groups.

\section{Haplotype analysis of S-locus genes in flowering Prunus taxa}

Considering the hybrid genome structure of Pyn, we performed comparative analysis of the S-locus between accessions of Pyn and the possible parental species distributed within approximately $3 \mathrm{~km}$ in a natural forest on Jeju Island (Fig. 6). The reference genome assembly of Pyn-Jeju2 included two S-locus haplotypes, named S1 and S2. The gene structure of the S1 haplotype was syntenic to that of Pp, consisting of S-RNase and SFB genes flanked by S-locus F box-like1 (SLFL1) and SLFL2. The S2 haplotype also showed the same order of genes except for SLFL2, which was not predicted across the 11-kb downstream region (Fig. 6a). Expression of $\mathrm{S}$-locus genes was characterized by tissue specificity. $S$-RNase and SFB genes were expressed only in pistil and stamen, respectively, with approximately twofold expression of S2 haplotype genes (Fig. 6b).

Using Illumina reads of wild Prunus accessions, we assembled the S-locus regions of four Pyn, three Ppa, two Pji, one Pjq, and one Psa accessions and compared them with those of the Pyn-Jeju2 reference genome. All the S-locus haplotypes of each accession were heterozygous with pairs of S-RNase and SFB genes, showing obligate out-crossing. Sequence comparison and phylogenetic analysis of $S$-RNase and $S F B$ genes in the S-locus regions classified $15 \mathrm{~S}$ haplotypes (Fig. 6c; Additional file 1: Figure S4). None of the accessions had the same combination of $\mathrm{S}$ haplotypes; however, six S haplotypes (S1, S3, S4, S8, S10, and S12) were shared among ten accessions. Of particular interest, each S haplotype from two Pyn accessions (Pyn-Jeju3 and Pyn-Jeju4) was directly linked to its counterpart in the genomes of one maternal (Ppa-3) and two paternal (Pjj-1 and Pjj-3) accessions. In contrast, paternal taxa shared none of the $\mathrm{S}$ haplotypes with maternal accessions. Comparison of the chloroplast genomes of the accessions supported the relationship between Pyn and Ppa in the maternal lineage (Additional file 12: Table S11). Overall, these findings suggest that the compatibility of S-locus haplotypes of maternal Ppa and paternal Pj could result in generation of Pyn as an inter-specific homoploid hybrid.

\section{Discussion}

In the research on plant genomes, high-quality reference genome assembly serves as a fundamental resource for 


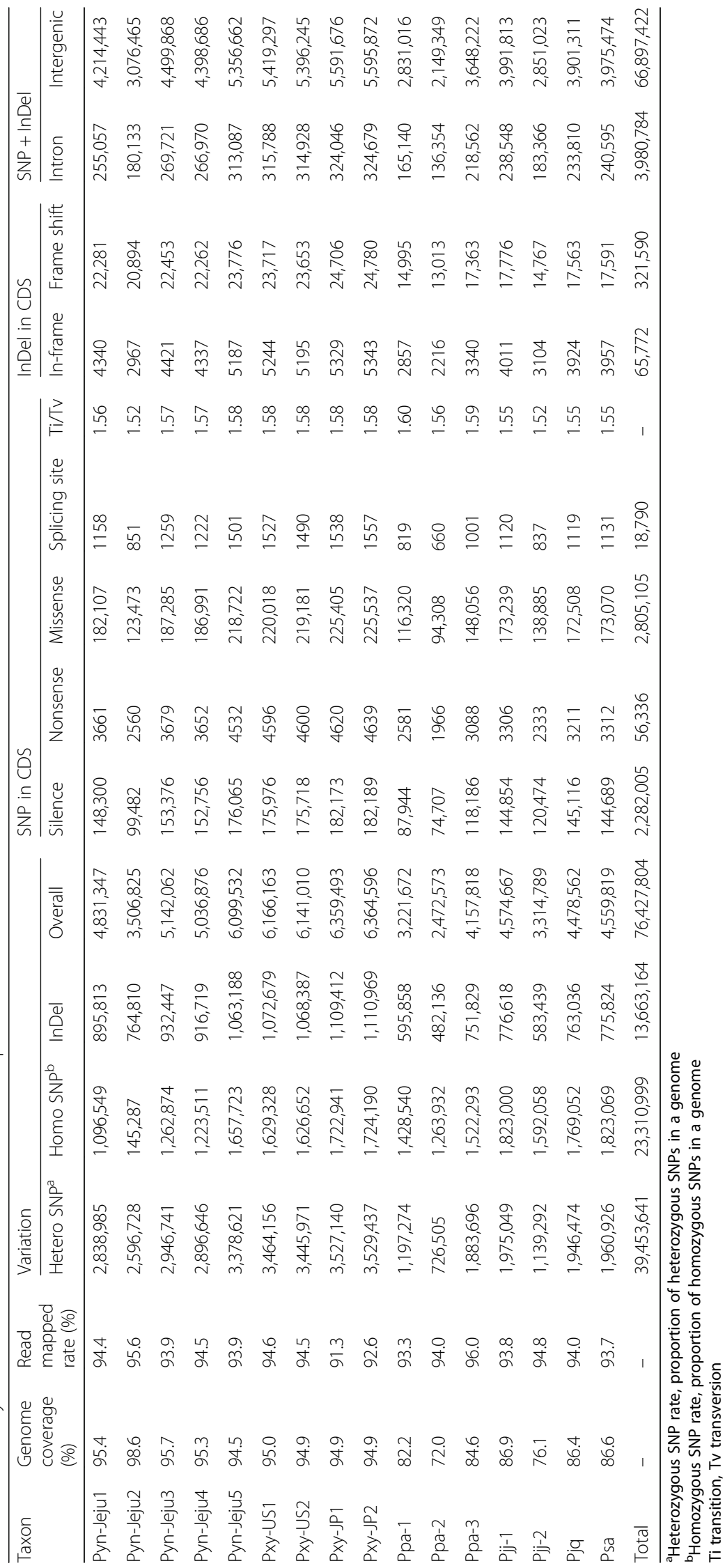


a

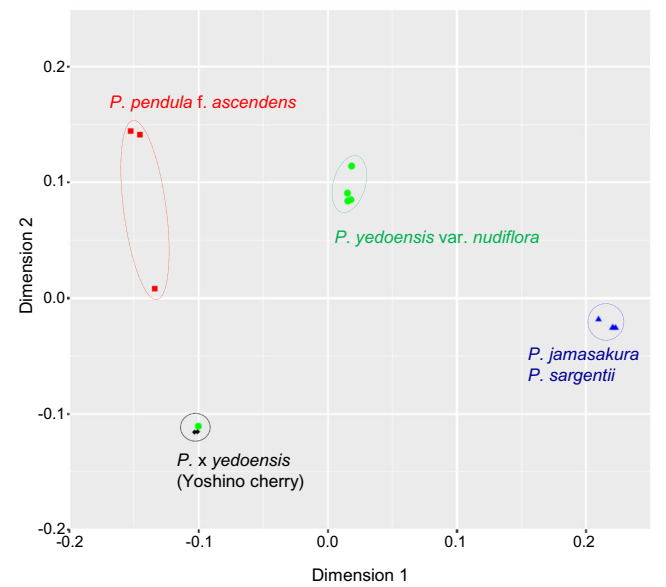

b

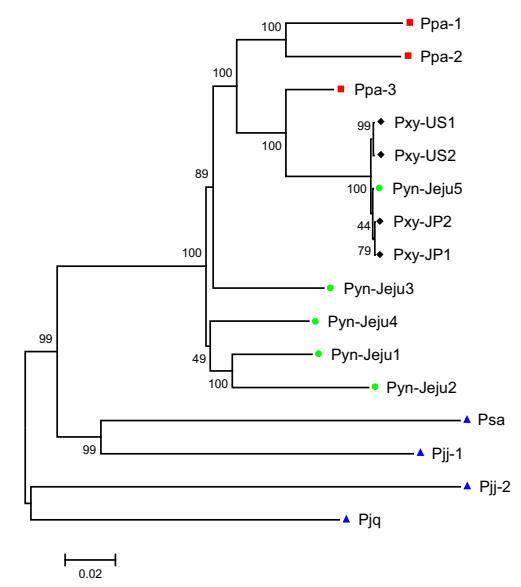

Fig. 5 The genomic relationships between flowering Prunus taxa. a Multidimensional scaling of Prunus accessions. Closely related accessions of Ppa (red square symbol), Pyn (green circle symbol), Pj or Psa (blue triangle symbol), and Pxy (black diamond symbol) are grouped together using dotted circles. b A maximum likelihood tree of Prunus accessions based on SNPs/InDels identified by variome analysis. The accession names are presented in Additional file 11: Table S10

a

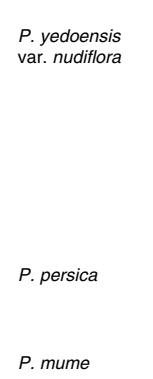

C

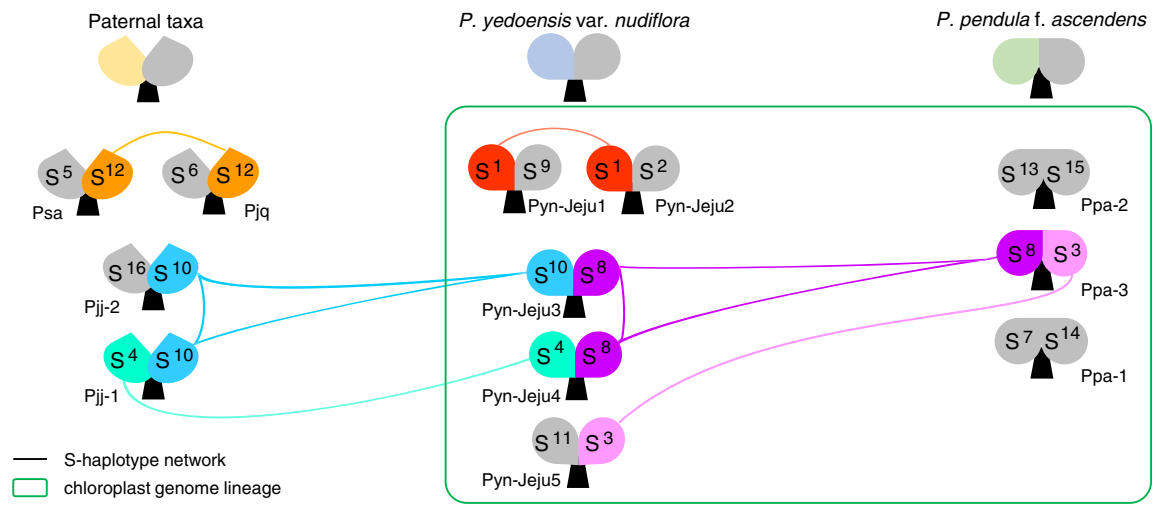

Fig. 6 Characterization of S haplotypes in flowering Prunus species. a Microsynteny at the S-locus regions between wild $P$. yedoensis and fruit crop Prunus ( $P$. persica and P. mume). There are two $S$ haplotypes in the heterozygous Pyn genome ( $31 \mathrm{~kb}$ of S1 and $35 \mathrm{~kb}$ of S2) compared to a single $\mathrm{S}$ haplotype in the homozygous Pp and Pm genomes. Syntenic genes are connected with lines. $\mathbf{b}$ Relative expression levels of the S-RNase and SFB genes in different tissues are presented by the average fragments per kilobase million (FPKM) value from three independent biological replicates. c S haplotype network in a natural Prunus population. A total of $15 \mathrm{~S}$ haplotypes from 12 accessions, which are distributed sympatrically in a natural habitat on Jeju Island, were identified. Accessions are placed according to their relative geographic location in the natural habitat. Shared $\mathbf{S}$ haplotypes between accessions are connected with lines of the same color. Chloroplast genome lineage, showing $<10$ nucleotide differences in the proteincoding sequences of the whole chloroplast DNA (Additional file 12: Table S11), is also presented in the green box 
various genomic studies. However, most assemblies of heterozygous genomes, such as out-bred, wild-type hybrid, and polyploid organisms, generated by short-read sequencing tend to produce a more fragmented assembly compared to homozygous genomes of similar size or complexity. Consequently, the utility and applications of heterozygous genomes with fragmented assemblies have been limited [29]. Approaches to developing an adequate solution for the sequencing and assembly of heterozygous genomes include sequencing both parents and offspring to infer haplotypes [30] and long-read sequencing combined with a phased diploid genome assembler [5].

A number of Prunus species have been identified as natural or artificial hybrids, many of which are fruit, nut, or ornamental crop species, providing genetic diversity in the Rosaceae family [15]. Hybrid Prunus species have been characterized either by phenotypic or genetic characteristics; however, no hybrid Prunus genomes have been characterized to date. In this study, we sequenced and analyzed the genome of Pyn, a hybrid flowering cherry, and provided sufficient information to solve the controversial issues surrounding this taxon, including parental origin, degree of hybridity, and taxon boundary against related species. Furthermore, this is the first report to provide valuable reference sequences for flowering cherry plants in the genus Prunus of the Rosaceae family for plant genomics and evolutionary analyses. More importantly, we successfully de novo assembled the heterozygous genome of a wild hybrid taxon. For the genome sequencing and assembly of Pyn, we used a long-read sequencing and assembly strategy combined with haplotype phasing using short-read sequences of putative parental species, Ppa and Pjj. The initial assembly was constructed using a FALCON assembler as "haplotype-fused" contigs and their associated heterozygous structural variants.

The overall assembly quality of the heterozygous genome was sufficiently high for the downstream analysis, including genome comparison, variome analysis, and expression profiling, on the basis of 4.2- and 481.9-fold longer N50 length of contiguous assembled sequences $(133 \mathrm{~kb})$ than that of the Pm (32 kb) and Pa (276 bp) genomes, respectively, which were assembled as a homozygous haploid genome using Illumina short-read sequences $[8,9]$. Genome level correspondence of the Pyn assembly to the eight chromosome pseudomolecules of $\mathrm{Pa}, \mathrm{Pm}$, and $\mathrm{Pp}$ also revealed the quality of the Pyn assembly. We anticipate that long-read sequencing and assembly is sufficiently effective to sequence, assemble, and analyze highly heterozygous wild plant genomes. Meanwhile, the assembly contiguity of Pyn was less than that of $\mathrm{Pp}(294 \mathrm{~kb})$, which was assembled based on Sanger sequencing data [10], presumably due to the absence of contig ordering and scaffolding by intensive genetic mapping. We included Illumina short-read PE and MP sequences as well as Fosmid-end sequences to order and scaffold the contigs; however, the assembly was not significantly improved. Therefore, we expect that the contiguity of long-read assembly for wild plants can be improved through the use of genome-wide chromatin interaction data such as $\mathrm{Hi}-\mathrm{C}$ [31].

It is noteworthy that the haplotype phasing of an assembly using putative parental species enabled us to investigate haplotype structures and the hybridity of Pyn. Phasing of genic regions of Pyn showed that approximately $41 \%$ of gene models were phased into maternal Ppa (19.4\%) and paternal Pjj (21.4\%) haplotypes. We further demonstrated that the Pyn accessions were grouped together with a similar distance to the Ppa and Pj groups by two dimensions in the MDS plot. Together with the almost identical chloroplast sequences of Pyn and Ppa accessions, these findings provide strong evidence supporting our previous suggestion that Pyn is likely an F1 hybrid taxon resulting from a maternal Ppa and paternal $\mathrm{Pj}$ cross. Of particular interest, Psa was clustered with Pjj and Pjq in both the MDS plot and the ML tree, suggesting that Pyn may have an additional source of paternal lineage compared to the single maternal lineage from Ppa. Additionally, the haplotype-specific genes showed abundant expression in various Pyn tissues. We assume that these lineage-specific genes, together with common genes originating from both parental lineages, may exert a synergistic effect to produce the superior ornamental characteristics of Pyn as a natural hybrid.

With respect to delimitation of the taxon boundary, genome-wide variome analysis using the Pyn assembly as a reference also provided a precise clue to the genetic relationship between Pyn and Pxy. Whole-genome resequencing, MDS plot, and ML tree analyses revealed that Pyn accessions were clearly separated from Pxy accessions. Pxy showed significant genome-level variation from Pyn and $\mathrm{Pj}$ accessions but showed a relatively close relationship to Ppa. This finding strongly suggested that Pyn and Pxy have a distinct paternal background. Interestingly, one accession of Pyn (Pyn-Jeju5) was tightly grouped with Pxy in both the MDS plot and the ML tree. Although this accession grows in a natural forest on Jeju Island, we assume that Pyn-Jeju5 is an accession of Pxy that escaped from the cultivated area. Considering the fact that Pyn-Jeju5 has been taxonomically classified as Pyn based on morphological characters, highly precise tools such as molecular markers should be utilized to correctly identify Pyn for evaluation and conservation of this taxon in nature.

For reproduction of Prunus species, successful pollination, fertilization, and seed formation are indispensable since parthenocarpic fruits have not developed in Prunus species. Our comparative genome analysis indicated that 
diversification of the Prunus genomes arose during the Paleocene (up to 66 Mya), followed by the successive split of Prunus species during the Eocene (36-44 Mya). This result is supported by the discovery of fossilized endocarps of $P$. wutuensis in eastern Asia dating to the Eocene [32]. Expansion of a specific category of genes related to auxin response and early flowering may have been involved in the diversification of flowering Prunus species. In addition, characterization of S-locus haplotypes, the determinant of Prunus GSI, in Pyn and its related species has yielded novel insights into the inter-specific hybridization of flowering Prunus in sympatric regions. All the flowering Prunus accessions investigated in this study had unique pairs of heterozygous $S$ haplotypes that suggested out-breeding. Nevertheless, two Pyn accessions retained a combination of parental S haplotypes, each of which was shared with one parental lineage, and several $\mathrm{S}$ haplotypes were also shared between accessions or even different taxa (Psa and Pjq). Considering the fact that these taxa are sympatrically distributed in a $3 \mathrm{~km}$ range on Jeju Island, and that their blooming time (late-March to mid-April) and flowering periods (approximately two weeks) overlap, we anticipate that cross pollination between closely related taxa resulted in a hybridization network of flowering Prunus in this natural habitat. These findings are consistent with our previous hypothesis that inter-specific hybridization and additional introgression by backcross between closely related flowering Prunus species on Jeju Island may produce a hybrid swarm [24]. Therefore, we anticipate that reproductive barriers between closely related flowering Prunus genomes are likely to be unestablished, presumably due to strong GSI, resulting in reduced restriction of inter-specific hybridization.

\section{Conclusions}

Hybridization has greatly increased plant diversity by generating new genetic combinations and genomes. However, the highly heterozygous genomic nature of hybrid species complicates genome studies due to the presence of highly similar sequences, a significant challenge in plant genomics. In this study, we successfully sequenced and assembled the draft genome of $P$. yedoensis var. nudiflora, a wild hybrid taxon of flowering Prunus, using long-read sequencing and a sequence phasing strategy. The results suggest that inter-specific hybridization due to the strong gametophytic self-incompatibility of flowering Prunus species may have contributed to the establishment of a natural hybrid taxon. The genome assembly of this taxon, along with the whole-genome resequencing data of related Prunus taxa, will provide valuable genomic resources for research, conservation, and breeding studies of Prunus species, benefiting both basic and applied plant biologists.

\section{Methods \\ Plant material and genome sequencing}

The No. 2 accession at the natural habitat of $P$. yedoensis var. nudiflora in Bongae-dong, Jeju Island (the Korea National Monument No. 159, Pyn-Jeju2; Fig. 1a) was chosen for genome sequencing. It was estimated to be 200 years old. Genomic DNA (gDNA) was extracted from young leaves collected in April 2015 using a DNeasy Plant Maxi Kit (Qiagen, Valencia, CA, USA) and then used for short-read sequencing. Separately, high molecular weight DNA was isolated according to the nuclei isolation method for single-molecule sequencing [33]. In total, $325.3 \mathrm{~Gb}$ of sequence data was obtained using Illumina and PacBio platforms (Additional file 2: Table S1) according to the manufacturer's protocols. For short-read sequencing, $205.5 \mathrm{~Gb}$ of Illumina sequences was generated using NextSeq and MiSeq (Illumina, San Diego, CA, USA) sequencing of 250-base pair (bp) (NextSeq) and 500-bp (MiSeq) insert libraries for PE sequencing and 3-, 5-, 10-, 15-, and 20-kb insert libraries for MP sequencing. In addition, one Fosmid library consisting of 55,200 clones with an average insert size of $40 \mathrm{~kb}(8.6 \times)$ was constructed using the NxSeq $40 \mathrm{~kb}$ Mate pair cloning kit (Lucigen, Middleton, WI, USA). Fosmid-end sequences were generated using HiSeq X Ten (Illumina, San Diego, CA, USA). Illumina reads from each library were collected at a minimum quality score $(\mathrm{Q} 20)$ and then filtered for adaptor contamination and low-quality regions using Trimmomatic v0.32 software [34]. Polymerase chain reaction (PCR) duplicates were removed by FastUniq v1.1 software [35] with a default parameter. Adaptor sequences and PCR duplicates of MP reads were filtered using NextClip software [36]. For single-molecule sequencing, $18.8 \mathrm{~Gb}$ of PacBio sequences was generated from a 20-kb library using a PacBio RSII sequencer (Pacific Biosciences, Menlo Park, CA, USA) and sequence errors were corrected using the $\mathrm{PBcR}$ pipeline from Celera Assembler 8.3.1 software [37] with default parameters. All of the sequence data generated in this study are summarized in Additional file 2: Table S1.

\section{Genome size estimation and de novo assembly}

The genome size of Pyn was estimated both by flow cytometry and K-mer analysis. For flow cytometry analysis, genome size was measured according to previous reports $[38,39]$ using a CyFlow Space system (Partec BmbH, Münster, Germany) and diploid Raphanus sativus cv. WK10039 $(1 \mathrm{C}=0.6 \mathrm{pg})$ as a reference. For K-mer analysis, the occurrences of K-mer with a peak depth were counted using Illumina PE reads, and genome size was calculated by dividing the total read length by coverage of K-mer peak using JELLYFISH 2.1.3 software [40] with 
K-mer 17. To assemble the sequence reads into a draft genome, a hierarchical hybrid assembly strategy was used (Additional file 1: Figure S2). First, PacBio reads were assembled into the initial scaffolds using the FALCON assembler v0.3.0 with parameters of length cutoff $12 \mathrm{~kb}$, max difference 100, max coverage 150, and minimum coverage 2 . The initial scaffolds were filtered with chloroplast and mitochondrial sequences using NUCmer in the MUMmer 3 package [41]. Next, Illumina reads were assembled with the chloroplast and mitochondria-filtered scaffolds to extend the scaffolds using SOAPdenovo2.04 software [42]. The resulting scaffolds were aligned with PacBio reads that had not been assembled into the initial scaffolds for additional assembly and gap filling using PBJelly 15.2.20 software [43] with minMatch 8 , minimum identity 70 , maxScore 500, noSplitSubreads, and support stage-capturedOnly parameters. Finally, draft assembly was improved by Pilon software [44] for additional gap filling and sequence error correction with fix bases, gaps, and diploid parameters.

\section{Genome annotation}

For gene prediction, a combination of ab initio and evidence-based gene predictions was used. The genome assembly was premasked first for class I and class II transposons using RepeatMasker 4.0.5 [45], RepeatModeler 1.0.8 [46], and LTR_FINDER v1.05 software [47], then protein-coding genes were predicted ab initio using BRAKER1 v1.8 [48], GlimmerHMM v3.0.2 [49], and SNAP [50] programs with parameters trained using the A. thaliana matrix. Genes with $<300$ bp of coding sequence or an incomplete coding region were filtered out. Predicted proteins with a top match to transposon-encoded proteins [51] and putative gene splits predicted on the unfinished gaps were also excluded from the annotation and gene counts. For evidence-based gene prediction, seven tissues, including floral bud, foliar bud, leaf, petal, stamen, pistil, and berry, were used to generate approximately 223.7 million filtered Illumina PE mRNA-seq reads (Additional file 3: Table S2). Total RNA was isolated from each tissue using the cetyl trimethylammonium bromide (CTAB) method [52], which was then used for mRNA purification, construction of a library with a 500-bp insert size, and sequencing according to the manufacturer's instructions. Quality filtering of mRNA-seq reads was performed according to our previous study [53]. The resulting transcript sequence data were aligned to the genome assembly and evidence-based gene sets were predicted using the PASA package [54]. We also aligned the gene models of Chinese plum $(\mathrm{Pm})$ [9], peach $(\mathrm{Pp})$ [10], strawberry (Fv) [13], apple (Mxd) [7], and A. thaliana (TAIR10) [55] to the genome assembly using
Exonerate 2.2.0 software [56]. EVidenceModeler software [54] was used to combine ab initio gene models, transcript alignments, and coding sequence alignments into consensus gene model sets. RNA genes were identified using Infernal [57] for tRNAs, BLASTN search for rRNAs, and sequence comparison using miRBase [58] for miRNAs. The predicted protein-coding genes were annotated based on SwissProt and TrEMBL databases from UniProt [59], RefSeq Plant, and nucleotide databases of the National Center for Biotechnology Information (NCBI) [60] using BLASTP with an E value cutoff of $1 \mathrm{E}^{-10}$ and query coverage of $70 \%$. The InterPro database [61] was also used to annotate motifs and domains and the gene ontology information for each gene model was extracted from InterPro.

\section{Transcriptome and comparative genome analysis}

Expression analysis of gene models in five tissues (leaf, petal, stamen, pistil, and berry) was performed using the mRNA-seq reads. PE reads were end-to-end aligned to the coding sequences (CDSs) of gene models using STAR 2.5.2b software [62] with default parameters. Reads that were mapped to multiple locations were excluded. The resulting mapped reads for each gene were normalized and patterns of gene expression between tissues were analyzed using DESeq2 [63] and MCLUST version 3 [64] that are included in the R/Bioconductor package. The data of three biological replicates were pooled and the average normalized read count values for genes were extracted and analyzed. Alternative splicing variants were analyzed using Cuffdiff2 [65] and spliceR [66]. A genome-wide synteny comparison between Pyn and $\mathrm{Pa}, \mathrm{Pm}$, or $\mathrm{Pp}$ was performed based on an all-against-all BLASTP comparison ( $\mathrm{E}$ value cutoff of $1 \mathrm{E}^{-10}$ ) and synteny regions were inferred using the MCScanX toolkit [27] with a match score of 50, match size of 5 , and gap penalty of -1 . Orthologous gene families among the Prunus genomes were identified using OrthoMCL v2.0 software [67] with all-against-all BLASTP ( $E$ value cutoff of $1 \mathrm{E}^{-5}$ and $>50 \%$ match) searches. Gene family comparison between the Prunus genomes was performed using the PLAZA 3.0 Dicots database [68]. Genome to genome synteny blocks of Pyn versus $\mathrm{Pp}, \mathrm{Pa}$, and $\mathrm{Pm}$ were plotted using an in-house perl script. For phylogenetic analysis of gene families, the amino acid sequences of orthologous genes were aligned using MUSCLE v3.8.31 software [69] with default parameters. Aligned sequences were trimmed at both ends using GBLOCKS $0.91 \mathrm{~b}$ software to eliminate regions of poor alignment [70]. Conserved blocks of aligned sequences were concatenated into a single sequence and phylogenetic trees were constructed using the ML method in MEGA7 software [71]. The stability of each tree node was tested by bootstrap analysis with 
1000 replicates. Ks between homologous genes was determined using the PAML package [72].

\section{Molecular dating}

To estimate the divergence time of the Prunus genomes, a total of 276 single copy orthologous genes, conserved in four Prunus species (Pyn, Pa, Pm, and Pp), two Rosaceae species (Mxd and $\mathrm{Fv}$ ), and one outgroup Fabaceae species (Mt), were selected using reciprocal BLASTP with an $\mathrm{E}$ value cutoff of $1 \mathrm{E}^{-10}$ and query coverage of $70 \%$. For Bayesian evolutionary analysis, BEAST v1.7 [73] was used. Input files were generated by BEAUti interface with a GTR $+\mathrm{I}+\mathrm{G}$ model and the combined dataset was applied for the BEAST analysis with a Yule speciation tree and an uncorrelated lognormal molecular clock model. We constrained the crown age of Rosaceae-Fabaceae with a uniform distribution from 100 to 107 Mya, following Moore et al. [74]. In addition, we applied two calibration points, following Chin et al. [28], each with a uniform distribution as follows: (1) the crown age of Rosaceae at 84.2-92.8 Mya; and (2) the crown age of Prunus at 51.6-65.2 Mya. Posterior distributions of parameters were approximated using two independent MCMC analyses of 20,000,000 generations with a $10 \%$ burn-in. The results were verified using Tracer v1.5 [75] to ensure that plots from the two analyses converged on the same area and then combined. The samples from the posterior analysis were summarized on a maximum clade credibility tree, which had the maximum sum of posterior probabilities on its internal nodes using TreeAnnotator v1.5.4 [73] with the posterior probability limit set to 0.5 . Means and $95 \%$ HPD of age estimates were obtained from the combined outputs using Tracer. The results were visualized using FigTree v1.4.2 [76].

\section{Resequencing, haplotype phasing, and variation analysis}

Five Pyn accessions from Jeju Island, Korea, four "Yoshino cherry" accessions (Pxy) from Tokyo, Japan, and Washington, DC, USA, and seven accessions of closely related species of Pyn, including Pji, Pjq, Ppa, and Psa, were selected for resequencing analysis (Additional file 11: Table S10). gDNA was extracted from the leaves of each accession as described above. Sequencing was performed by Illumina MiSeq, NextSeq or HiSeq (Illumina, San Diego, CA, USA) PE sequencing of libraries with 500 -bp inserts according to the manufacturer's protocol. At least $7.5 \times$ coverage of sequences was generated for each accession. All reads were preprocessed as mentioned above for quality. The genome size of each plant was calculated using the filtered reads as described above based on K-mer 17. For variome analysis, the filtered reads were aligned to the draft Pyn assembly using BWA-MEM 0.7.12 software [77] with a parameter of M. Duplicate alignments were removed using the MarkDuplicates module in the Picard 2.2.4 package [78]. Realignment around short InDels and SNP genotyping were performed using RealignerTargetCreator, IndelRealigner, and HaplotypeCaller modules in GATK 3.7 software [79]. Annotation of SNPs and InDels was performed using SnpEff 4.3p software [80]. For haplotype phasing of SNPs, SNP positions with a minimum depth of two reads were considered. BEDTools 2.25 software [81] was used to compute the alignment coverage of maternal (Ppa) and paternal (Pji) reads to the reference sequences. To determine the phased haplotype of SNPs and to impute missing genotype calls for the draft genome sequences, BEAGLE v4.0 software [82] was used under default parameters. For further analysis of genic region, genes were phased into one of the parental types if a gene was aligned only by reads from one parent species or had at least twofold as many supports for SNPs by reads of one parental species. Otherwise, genes were defined as common type. MDS for two dimensions based on a pairwise distance matrix between two different genotypes was performed by PLINK software [83] with the parameter of -genome, -cluster, -mds-plot 2. The MDS plots were drawn using R script. MEGA7 [71] was used to draw an ML tree based on a pairwise distance matrix of SNPs, which was calculated by counting the total number of different alleles between genotypes.

\section{Additional files}

Additional file 1: Figure S1. K-mer plots of wild $P$. yedoensis (Pyn) and "Yoshino cherry" (Pxy) accessions. Figure S2. A workflow of genome assembly and annotation. Figure S3. Chromosomal comparison of the gene-phased genome assembly of wild $P$. yedoensis (Pyn) with the $P$. avium $(\mathrm{Pa})$ and $P$. mume $(\mathrm{Pm})$ genomes. Figure S4. A maximum likelihood tree of S-locus genes showing the phylogenetic relationship among the $S$ haplotypes. (DOCX $670 \mathrm{~kb}$ )

Additional file 2: Table S1. Statistics of genome sequence data of wild $P$. yedoensis (Pyn-Jeju2) used in this study. (XLSX $11 \mathrm{~kb}$ )

Additional file 3: Table S2. Statistics of transcriptome sequence data of wild P. yedoensis (Pyn-Jeju2) used in this study. (XLSX 11 kb)

Additional file 4: Table S3. Evaluation of gene space coverage of the wild $P$. yedoensis genome using transcriptome unigenes. (XLSX $10 \mathrm{~kb}$ )

Additional file 5: Table S4. Summary of repetitive sequences identified in the draft genome of wild P. yedoensis. (XLSX $12 \mathrm{~kb}$ )

Additional file 6: Table S5. Statistics of gene models predicted from the draft genome of wild $P$. yedoensis. (XLSX $10 \mathrm{~kb}$ )

Additional file 7: Table S6. Annotation statistics of the wild $P$. yedoensis gene set. (XLSX $10 \mathrm{~kb}$ )

Additional file 8: Table S7. Summary of alternative splicing events identified in protein-coding genes. (XLSX $10 \mathrm{~kb}$ )

Additional file 9: Table S8. Coverage of individual chromosomes of peach $(\mathrm{Pp})$, sweet cherry $(\mathrm{Pa})$, and Chinese plum $(\mathrm{Pm})$ showing synteny with the counterpart of wild $P$. yedoensis (Pyn) genome. (XLSX 11 kb) 
Additional file 10: Table S9. Over- or under-represented gene families in the wild $P$. yedoensis genome compared to the $P$. avium, $P$. mume, and P. persica genomes. (XLSX $14 \mathrm{~kb})$

Additional file 11: Table S10. Summary of accessions and Illumina short-read data used in whole-genome resequencing analysis. (XLSX 11 $\mathrm{kb})$

Additional file 12: Table S11. Comparison of the chloroplast genomes between Prunus accessions. (XLSX $10 \mathrm{~kb}$ )

\section{Abbreviations}

bp: Base pair; CDS: Coding sequence; CTAB: Cetyl trimethylammonium bromide; DEG: Differentially expressed gene; FPKM: Fragments per kilobase million; Fv: Fragaria vesca; Gb: Gigabase; gDNA: Genomic DNA; GSI: Gametophytic self-incompatibility; HPD: Higher posterior density; InDel: Insertion or deletion; Kb: Kilobase; K-mer: Substring of length K; Ks: Synonymous substitution rate; Mb: Megabase; MDS: Multidimensional scaling; ML: Maximum likelihood; MP: Mate-paired; mRNA-seq: Messenger RNA sequencing; Mt: Medicago truncatula; Mxd: Malus x domestica; Mya: Million years ago; NCBI: National Center for Biotechnology Information; NGS: Next-generation sequencing; PCR: Polymerase chain reaction; PE: Paired end; Pjj: P. jamasakura var. jamasakura; Pjq: P. jamasakura var. quelpaertensis; Ppa: $P$. pendula f. ascendens; Psa: $P$. sargentii; Pxy: $P$. x yedoensis; Pyn: $P$. yedoensis var. nudiflora; SFB: S haplotype-specific F-box protein; SNP: Single nucleotide polymorphism; S-RNase: S-locus ribonuclease; Ti: Transition; Tv: Transversion

\section{Acknowledgements}

We thank Dr. Chan-Soo Kim and Dr. Youngje Kang of Warm Temperate and Subtropical Forest Research Center, National Institute of Forest Science for collecting plant samples on Jeju Island, Korea. We also thank Dr. Jin Murata and Dr. Tetsuo Toma of Koishikawa Botanic Garden and Dr. Toshio Katsuki of Tama Forest Science Garden, FFPRI, Japan for collecting Yoshino cherry.

\section{Funding}

This work was mainly supported by grants from the Scientific Research program (KNA1-1-13 and 14-1) of Korea National Arboretum and partly by a grant from the Next-Generation Biogreen21 program (PJ013194), Rural Development Administration, Korea.

\section{Availability of data and materials}

The assembled sequences as well as sequence reads described in this study have been deposited in the National Center for Biotechnology and Information (NCBI) under the accession PJQY00000000 [84] and SRP136962 [85]. Gene models of Rosaceae species used in genome comparison are available from Velasco et al. [7], Shirasawa et al. [8], Zhang et al. [9], The International Peach Genome Initiative [10], and Shulaev et al. [13].

\section{Authors' contributions}

JHM planned the projects, designed the research, analyzed data, and wrote the manuscript. SB performed the experiments, analyzed data, and wrote the manuscript. KC and JHK planned the projects and participated in manuscript preparation. GBK, HJY, AC, HJ, CK, HJK, and KSC participated in plant sampling, sequencing, data analysis, and manuscript preparation. All authors read and approved the final manuscript.

\section{Ethics approval and consent to participate}

Wild tree samples were provided from the Korea National Arboretum. In addition, the authors obtained permission for plant sampling from Korea National Arboretum.

\section{Competing interests}

The authors declare that they have no competing interests.

\section{Publisher's Note}

Springer Nature remains neutral with regard to jurisdictional claims in published maps and institutional affiliations.

\section{Author details}

'Department of Bioscience and Bioinformatics, Myongji University, Yongin

17058, Korea. ${ }^{2}$ Korea National Arboretum, Pocheon 11186, Korea.

${ }^{3}$ Department of Life Science, The Catholic University of Korea, Bucheon

14662, Korea. ${ }^{4}$ Department of Life Science, Gachon University, Seongnam 13120, Korea.

Received: 18 January 2018 Accepted: 1 August 2018

Published online: 04 September 2018

\section{References}

1. Wendel J, Jackson S, Meyers B, Wing R. Evolution of plant genome architecture. Genome Biol. 2016;17:37.

2. Soltis $\mathrm{P}$, Soltis D. The role of hybridization in plant speciation. Annu Rev Plant Biol. 2009;60:561-88.

3. Koren S, Phillippy A. One chromosome, one contig: complete microbial genomes from long-read sequencing and assembly. Curr Opin Microbiol. 2015:23:110-20.

4. Jiao $W-B$, Schneeberger $K$. The impact of third generation genomic technologies on plant genome assembly. Curr Opin Plant Biol. 2017;36:64-70.

5. Chin C, Peluso P, Sedlazeck F, Nattestad M, Concepcion G, Clum A, et al. Phased diploid genome assembly with single-molecule real-time sequencing. Nat Metohods. 2016;13:1050-4.

6. Potter D, Eriksson T, Evans R, Oh S, Smedmark J, Morgan D, et al. Phylogeny and classification of Rosaceae. PI Syst Evol. 2007;266:5-43.

7. Velasco R, Zharkikh A, Affourtit J, Dhingra A, Cestaro A, Kalyanaraman A, et al. The genome of the domesticated apple (Malus $\times$ domestica Borkh.). Nat Genet. 2010;42:833-9.

8. Shirasawa K, Isuzugawa K, Ikenaga M, Saito Y, Yamamoto T, Hirakawa H, et al. The genome sequence of sweet cherry (Prunus avium) for use in genomics-assisted breeding. DNA Res. 2017;24:499-508.

9. Zhang Q, Chen W, Sun L, Zhao F, Huang B, Yang W, et al. The genome of Prunus mume. Nat Commun. 2012;3:1318. https://www.ncbi.nlm.nih.gov/ bioproject/171605. Accessed 10 Sept 2016

10. The International Peach Genome Initiative. The high-quality draft genome of peach (Prunus persica) identifies unique patterns of genetic diversity, domestication and genome evolution. Nat Genet. 2013:45:487-94.

11. Wu J, Wang Z, Shi Z, Zhang S, Ming R, Zhu S, et al. The genome of the pear (Pyrus bretschneideri Rehd.). Genome Res. 2013;23:396-408.

12. Chagné $D$, Crowhurst $R$, Pindo $M$, Thrimawithana A, Deng $C$, Ireland $H$, et al. The draft genome sequence of European pear (Pyrus communis L. 'Bartlett'). PLoS One. 2014;9:e92644.

13. Shulaev V, Sargent D, Crowhurst R, Mockler T, Folkerts O, Delcher A, et al. The genome of woodland strawberry (Fragaria vesca). Nat Genet. 2011;43:109-16.

14. Hirakawa H, Shirasawa K, Kosugi S, Tashiro K, Nakayama S, Yamada M, et al. Dissection of the octoploid strawberry genome by deep sequencing of the genomes of Fragaria species. DNA Res. 2014;21:169-81.

15. Ma H, Olsen R, Pooler M, Kramer M. Evaluation of flowering cherry species, hybrids, and cultivars using simple sequence repeat markers. J Amer Soc Hort Sci. 2009:134:435-44.

16. Knight R. Abstract bibliography of fruit breeding and genetics to 1965; Prunus. Farnham Royal: Commonwealth Agricultural Bureau; 1969.

17. Takenaka Y. The origin of the Yoshino cherry tree. J Hered. 1963;54:207-11.

18. Koehne VE. 95 Prunus yedoensis var. nudiflora, nov. var. Von E. Koehne. Repertorium Specierum Novarum Regni Vegetabilis. 1912;10:507.

19. Jung $\mathrm{Y}-\mathrm{H}$, Oh M-Y. Phylogenetic relationships of Prunus (Rosaceae) in Korea and Japan inferred from chloroplast DNA sequences. Kor J Genet. 2005;27: 279-88.

20. Roh M, Cheong E, Choi I-Y, Young Y. Characterization of wild Prunus yedoensis analyzed by inter-simple sequence repeat and chloroplast DNA. Sci Hortic. 2007:114:121-8.

21. Sassa H, Kakui H, Minamikawa M. Pollen-expressed F-box gene family and mechanism of S-RNase-based gametophytic self-incompatibility (GSI) in Rosaceae. Sex Plant Reprod. 2010;23:39-43.

22. Vieira J, Santos R, Habu T, Tao R, Vieira C. The Prunus self-incompatibility locus (S locus) is seldom rearranged. J Hered. 2008;99:657-60.

23. Cho M, Kim C, Kim S, Kim T, Heo K, Jun J, et al. Molecular and morphological data reveal hybrid origin of wild Prunus yedoensis (Rosaceae) from Jeju Island, Korea: implications for the origin of the flowering cherry. Am J Bot. 2014;101:1976-86. 
24. Cho A, Baek S, Kim G-B, Shin C-H, Kim C-S, Choi K, et al. Genomic clues to the parental origin of the wild flowering cherry Prunus yedoensis var. nudiflora (Rosaceae). Plant Biotechnol Rep. 2017;11:449-59.

25. Kim S-Y, Kim M-H, Kim J. The chromosome index of Korean native plants. Incheon: National Institute of Biological Resources; 2012.

26. Plant DNA. C-value database. http://data.kew.org/cvalues. Accessed 10 Mar 2016.

27. Wang Y, Tang H, DeBarry J, Tan X, Li J, Wang X, et al. MCScanX: a toolkit for detection and evolutionary analysis of gene synteny and collinearity. Nucleic Acids Res. 2012;40:e49.

28. Chin S-W, Shaw J, Haberle R, Wen J, Potter D. Diversification of almonds, peaches, plums and cherries - molecular systematics and biogeographic history of Prunus (Rosaceae). Mol Phylogenet Evol. 2014;76:34-48.

29. Alkan C, Sajjadian S, Eichler E. Limitations of next-generation genome sequence assembly. Nat Mathods. 2011:8:61-5.

30. Xu Q, Chen L, Ruan X, Chen D, Zhu A, Chen C, et al. The draft genome of sweet orange (Citrus sinensis). Nat Genet. 2013;45:59-66.

31. Ghurye J, Pop M, Koren S, Bickhart D, Chin C. Scaffolding of long read assemblies using long range contact information. BMC Genomics. 2017;18:527.

32. Li Y, Smith T, Liu C-J, Awasthi N, Yang J, Wang Y-F, et al. Endocarps of Prunus (Rosaceae: Prunoideae) from the early Eocene of Wutu, Shandong Province, China. Taxon. 2011;60:555-64.

33. Zhang M, Zhang Y, Scheuring C, Wu C, Dong J, Zhang H. Preparation of megabase-sized DNA from a variety of organisms using the nuclei method for advanced genomics research. Nat Protoc. 2012;7:467-78.

34. Bolger A, Lohse M, Usadel B. Trimmomatic: a flexible trimmer for Illumina sequence data. Bioinformatics. 2014;30:2114-020.

35. Xu H, Luo X, Qian J, Pang X, Song J, Qian G, et al. FastUniq: a fast de novo duplicates removal tool for paired short reads. PLoS One. 2012;7:e52249.

36. Leggett R, Clavijo B, Clissold L, Clark M, Caccamo M. NextClip: an analysis and read preparation tool for Nextera long mate pair libraries. Bioinformatics. 2014;30:566-8

37. Berlin K, Koren S, Chin C, Drake J, Landolin J, Phillippy A. Assembling large genomes with single-molecule sequencing and locality-sensitive hashing. Nat Biotechnol. 2015;33:623-30.

38. Hwang Y-J, Yu H-J, Mun J-H, Ryu K, Park B-S, Lim K-B. Centromere repeat DNA originated from Brassica rapa is detected in the centromere region of Raphanus sativus chromosomes. Kor J Hort Sci Technol. 2012;30:751-6.

39. Dolezel J, Bartos J, Voglmayr H, Greilhuber J. Nuclear DNA content and genome size of trout and human. Cytometry A. 2003;51:127-8.

40. Marçais $G$, Kingsford C. A fast, lock-free approach for efficient parallel counting of occurrences of k-mers. Bioinformatics. 2011;27:764-70.

41. Kurtz S, Phillippy A, Delcher A, Smoot M, Shumway M, Antonescu C, et al. Versatile and open software for comparing large genomes. Genome Biol. 2004;5:R12.

42. Luo R, Liu B, Xie Y, Li Z, Huang W, Yuan J, et al. SOAPdenovo2: an empirically improved memory-efficient short-read de novo assembler. Gigascience. 2012;1:18.

43. English A, Richards S, Han Y, Wang M, Vee V, Qu J, et al. Mind the gap: upgrading genomes with Pacific biosciences RS long-read sequencing technology. PLoS One. 2012;7:e47768.

44. Walker B, Abeel T, Shea T, Priest M, Abouelliel A, Sakthikumar S, et al. Pilon: an integrated tool for comprehensive microbial variant detection and genome assembly improvement. PLoS One. 2014;9:e112963.

45. RepeatMasker 4.0.5. http://www.repeatmasker.org. Accessed 24 June 2016.

46. RepeatModeler. http://www.repeatmasker.org. Accessed 24 June 2016.

47. Xu Z, Wang H. LTR_FINDER: an efficient tool for the prediction of full-length LTR retrotransposons. Nucleic Acids Res. 2007;35:W265-8.

48. Hoff K, Lange S, Lomsadze A, Borodovsky M, Stanke M. BRAKER1: unsupervised RNA-Seq-based genome annotation with GeneMark-ET and AUGUSTUS. Bioinformatics. 2016;32:767-9.

49. Majoros W, Pertea M, Salzberg S. TigrScan and GlimmerHMM: two open source ab initio eukaryotic gene-finders. Bioinformatics. 2004;20:2878-9.

50. Korf I. Gene finding in novel genomes. BMC Bioinformatics. 2004:5:59.

51. Nussbaumer T, Martis M, Roessner S, Pfeifer M, Bader K, Sharma S, et al. MIPS PlantsDB: a database framework for comparative plant genome research. Nucleic Acids Res. 2013;41:D1144-51.

52. Chang S, Puryear J, Cairney J. A simple and efficient method for isolating RNA from pine trees. Plant Mol Biol Rep. 1993;11:113-6.

53. Jeong Y-M, Kim N, Ahn B, Oh M, Chung W, Chung H, et al. Elucidating the triplicated ancestral genome structure of radish based on chromosome-level comparison with the Brassica genomes. Theor Appl Genet. 2016;129:1357-72.
54. Haas B, Salzberg S, Zhu W, Pertea M, Allen J, Orvis J, et al. Automated eukaryotic gene structure annotation using EVidenceModeler and the program to assemble spliced alignments. Genome Biol. 2008;9:R7.

55. The Arabidopsis Genome Initiative. Analysis of the genome sequence of the flowering plant Arabidopsis thaliana. Nature. 2000; 408:796-815.

56. Slater G, Birney E. Automated generation of heuristics for biological sequence comparison. BMC Bioinformatics. 2005;6:31.

57. Nawrocki E, Eddy S. Infernal 1.1: 100-fold faster RNA homology searches. Bioinformatics. 2013;29:2933-5

58. Griffiths-Jones S, Saini H, van Dongen S, Enright AJ. miRBase: tools for microRNA genomics. Nucleic Acid Res. 2008;36:D154-8.

59. UniProt. http://www.ebi.ac.uk/uniprot. Accessed 16 Dec 2016.

60. The National Center for Biotechnology Information. www.ncbi.nlm.nih.gov.

61. InterPro. http://www.ebi.ac.uk/interpro. Accessed 16 Dec 2016.

62. Dobin A, Davis C, Schlesinger F, Drenkow J, Zaleski C, Jha S, et al. STAR: ultrafast universal RNA-seq aligner. Bioinformatics. 2013; 29:15-21.

63. Love M, Huber W, Anders S. Moderated estimation of fold change and dispersion for RNA-seq data with DESeq2. Genome Biol. 2014;15:550.

64. Fraley C, Raftery A. MCLUST version 3: an R package for normal mixture modeling and model-based clustering. Technical report no 504 Department of Statistics. Seattle: University of Washington; 2006.

65. Trapnell C, Hendrickson D, Sauvageau M, Goff L, Rinn J, Pachter L. Differential analysis of gene regulation at transcript resolution with RNA-seq. Nat Biotechnol. 2013;31:46-53.

66. Vitting-Seerup K, Porse B, Sandelin A, Waage J. spliceR: an R package for classification of alternative splicing and prediction of coding potential from RNA-seq data. BMC Bioinformatics. 2014;15:81.

67. Fischer S, Brunk B, Chen F, Gao X, Harb O, lodice JB, et al. Using OrthoMCL to assign proteins to OrthoMCL-DB groups or to cluster proteomes into new ortholog groups. Curr Protoc Bioinformatics. 2011;35:6.12.1-16.12.19.

68. Proost $S$, Van Bel M, Vaneechoutte D, Van de Peer Y, Inzé D, Mueller-Roeber B, et al. PLAZA 3.0: an access point for plant comparative genomics. Nucleic Acid Res. 2015;43:D974-81

69. Edgar R. MUSCLE: multiple sequence alignment with high accuracy and high throughput. Nucleic Acids Res. 2004;32:1792-7.

70. Talavera G, Castresana J. Improvement of phylogenies after removing divergent and ambiguously aligned blocks from protein sequence alignments. Syst Biol. 2007:56:564-77.

71. Kumar S, Stecher G, Tamura K. MEGA7: molecular evolutionary genetics analysis version 7.0 for bigger datasets. Mol Biol Evol. 2016;33:1870-4.

72. Yang Z. PAML 4: phylogenetic analysis by maximum likelihood. Mol Biol Evol. 2007;24:1586-91.

73. Drummond A, Rambaut A. BEAST: Bayesian evolutionary analysis by sampling trees. BMC Evol Biol. 2007;7:214.

74. Moore M, Soltis P, Bell C, Burleigh J, Soltis D. Phylogenetic analysis of 83 plastid genes resolves relationships among major clades of eudicot angiosperms and reveals multiple rapid radiations. Proc Natl Acad Sci U S A 2010;107:4623-8.

75. Tracer. http://tree.bio.ed.ac.uk/software/tracer. Accessed 2 Apr 2018.

76. FigTree. http://tree.bio.ed.ac.uk/software/figtree. Accessed 2 Apr 2018

77. Aligning sequence reads, clone sequences and assembly contigs with BWAMEM. https://arxiv.org/pdf/1303.3997v2.pdf. Accessed 24 Aug 2017.

78. Picard. http://broadinstitute.github.io/picard. Accessed 24 Aug 2017.

79. McKenna A, Hanna M, Banks E, Sivachenko A, Cibulskis K, Kernytsky A, et al. The genome analysis toolkit: a MapReduce framework for analyzing nextgeneration DNA sequencing data. Genome Res. 2010;20:1297-303.

80. Cingolani P, Platts A, Wang L, Coon M, Nguyen $T$, Wang $L$, et al. A program for annotating and predicting the effects of single nucleotide polymorphisms, SnpEff: SNPs in the genome of Drosophila melanogaster strain w1118; iso-2; iso-3. Fly (Austin). 2012;6:80-92.

81. Quinlan A, Hall I. BEDTools: a flexible suite of utilities for comparing genomic features. Bioinformatics. 2010;26:841-2.

82. Browning S, Browning B. Rapid and accurate haplotype phasing and missing-data inference for whole-genome association studies by use of localized haplotype clustering. Am J Hum Genet. 2007;81:1084-97.

83. Purcell S, Neale B, Todd-Brown $K$, Thomas L, Ferreira M, Bender D, et al. PLINK: a tool set for whole-genome association and population-based linkage analyses. Am J Hum Genet. 2007:81:559-75.

84. Baek S, Choi K, Kim G, Yu H, Cho A, Jang H, et al. Draft genome sequence of wild Prunus yedoensis reveals massive inter-specific hybridization between sympatric flowering cherries. 2018. https://www.ncbi.nlm.nih.gov/search/ 
?term=PJQY00000000\&utm_expid=.fAeHyO5JTBGxnObh2WlrCA.0\&utm_ referrer=https\%3A\%2F\%2Fwww.ncbi.nlm.nih.gov\%2F.

85. Baek S, Choi K, Kim G, Yu H, Cho A, Jang H, et al. Draft genome sequence of wild Prunus yedoensis reveals massive inter-specific hybridization between sympatric flowering cherries. 2018. https://www.ncbi.nlm.nih.gov/search/ ?term=SRP136962\&utm_expid=.fAeHyO5JTBGxnObh2WlrCA.0\&utm_referrer= https\%3A\%2F\%2Fwww.ncbi.nlm.nih.gov\%2F.

Ready to submit your research? Choose BMC and benefit from:

- fast, convenient online submission

- thorough peer review by experienced researchers in your field

- rapid publication on acceptance

- support for research data, including large and complex data types

- gold Open Access which fosters wider collaboration and increased citations

- maximum visibility for your research: over $100 \mathrm{M}$ website views per year

At BMC, research is always in progress.

Learn more biomedcentral.com/submissions 